\title{
Human vaccine and immunisation research in South Africa: A bibliometric study
}

\author{
M I Majiet, ${ }^{1} \mathrm{MB}$ ChB (5th-year student); E C Haddison, ${ }^{2} \mathrm{MD}$, MPH; E Amponsah-Dacosta, ${ }^{3} \mathrm{PhD}, \mathrm{MPH}$; G D Hussey, ${ }^{3,4} \mathrm{MB}$ ChB, \\ MMed (Public Health), MSc, FFCH, DTM\&H; R Muloiwa, ${ }^{3,5}$ MB ChB, DCH (SA), FCPaed (SA), MSc, PhD; B M Kagina, ${ }^{3}$ MSc, PhD \\ ${ }^{1}$ Faculty of Health Sciences, University of Cape Town, South Africa \\ ${ }^{2}$ Saa District Health Service, Centre Regional Delegation of Public Health, Saa, Cameroon \\ ${ }^{3}$ Vaccines for Africa Initiative (VACFA), School of Public Health and Family Medicine, Faculty of Health Sciences, University of Cape Town, \\ South Africa \\ ${ }^{4}$ Division of Medical Microbiology, Institute of Infectious Diseases and Molecular Medicine, Faculty of Health Sciences, University of Cape Town, \\ South Africa \\ ${ }^{5}$ Department of Paediatrics and Child Health, Faculty of Health Sciences, Groote Schuur Hospital and University of Cape Town, South Africa
}

Corresponding author: $M$ I Majiet (mjtmog005@myuct.ac.za)

Background. Research in human vaccines and immunisation plays a crucial role in shaping national, regional and global health policies aimed at controlling vaccine preventable diseases (VPDs). To our knowledge, the landscape of human vaccine and immunisation research in South Africa (SA) is not well characterised.

Objectives. To characterise research in human vaccines and immunisation in SA.

Methods. We conducted a bibliometric study. Seven electronic databases (PubMed; Scopus; Web of Science; Cochrane; CINAHL; Africa-Wide Information; and MEDLINE (via EBSCOhost)) were searched for eligible studies published in English between 1 January 2007 and 31 March 2020. Selected primary studies needed to be on human vaccine and immunisation research conducted in SA. All types of reviews were excluded. For the included studies, outcomes of interest included publication journals, publication trends, types of studies and VPDs, targeted populations, as well as author affiliations.

Results. A total of 9212 studies was retrieved. After screening for eligibility, 366 studies met the inclusion criteria. The key findings were as follows: ( $i$ ) a total of 54 (14.8\%) articles on human vaccine and immunisation research in SA appeared in local journals, while 312 (85.2\%) were in non-SA (international) journals; (ii) the number of publications on human vaccine and immunisation research in SA increased from 13 in 2007 to 47 in 2015; (iii) there were 189 (51.7\%) operational studies and 177 (48.3\%) clinical studies, with 88 (49.7\%) of the latter being clinical trials; (iv) human vaccine and immunisation research in SA is conducted across all age groups, with a focus on children; and $(v)$ authors of the identified research outputs and those mostly represented were from the universities of Cape Town and the Witwatersrand, Johannesburg. Conclusions. The landscape of human vaccine and immunisation research in SA is growing and adapting to the emerging trends in vaccinology, with a focus on the duo epidemic of HIV and TB, as well as Expanded Programme on Immunisation (EPI)-related vaccinations. This research contributes to locally relevant evidence that can be used to inform future vaccine and EPI-related research.

S Afr Med J 2021;111(7):649-654. https://doi.org/10.7196/SAMJ.2021.v111i7.15120

Since the Expanded Programme on Immunisation (EPI) was introduced in 1974, immunisation has played a significant role in reducing childhood morbidity and mortality caused by vaccine preventable diseases (VPDs) globally. ${ }^{[1]}$

To advance and maximise the public health benefits of immunisation at national level, the 2011 - 2020 Global Vaccine Action Plan (GVAP) outlined its first strategy as getting all countries commit to immunisation as a priority. ${ }^{[2]}$ Countries' commitment should go hand in hand with ownership, although this is not always the case. Closely linked with the country's immunisation ownership is the presence of a national immunisation technical advisory group (NITAG) ${ }^{[2]}$ NITAG is a multidisciplinary group of national experts who independently advise national immunisation programmes (NIPs) on matters related to vaccines and immunisation. ${ }^{[3]}$ The advice provided by NITAGs must be evidence based and locally relevant. Therefore, vaccine and immunisation research is critical in building the evidence base that NITAGs use to advise NIPs.

As with the 2011 - 2020 GVAP, the core principle of the World Health Organization (WHO) Immunisation Agenda 2030 is to be people focused, country owned, partnership based and data enabled. Considering this, there is a need for immunisation data that highlight critical gaps in vaccine research. Such data could contribute to advancing the research agenda to build an evidence base and support NIPs. ${ }^{[4]}$ It is also worth noting that the emergence of the COVID-19 pandemic further emphasises the need for appropriate evidence generation to inform national immunisation practices and policies during crises.

Globally, the immunisation landscape has significantly evolved since the establishment of EPI in 1974. As EPI developed and expanded into low- and middle-income countries (LMICs), more information was required to inform decision and implement policies.

A previous study investigating vaccine research in Africa between 1970 and 2010, reported an uneven distribution of research outputs across countries. Of the research included in the study, 6 countries accounted for over half of the research outputs. The authors identified the need for development of research capacity in LMICs in general. ${ }^{[5]}$ Mashingaidze et al.' $\mathrm{s}^{[5]}$ study was later updated for 2011 - 2017. The findings from the updated study ${ }^{[6]}$ alluded to vastly increased research 
outputs driven by the burden of disease on the continent and a need to address the lack of finance and policy-related research that had also been present in the previous study. Vaccine and immunisation research from SA featured prominently in Machingaidze et al.'s ${ }^{[5]}$ study and the updated study. ${ }^{[6]}$ We were therefore interested in a more detailed characterisation of SA human vaccine and immunisation research.

The immunisation landscape has significantly evolved since the establishment of EPI in SA (EPI-SA). This is evidenced by the evolution of the vaccination schedule and the establishment of the National Advisory Group on Immunisation (NAGI) in 1993 to advise the National Department of Health (DoH) on issues related to vaccination. ${ }^{[7]}$ For example, the first vaccines introduced into EPI-SA were for diphtheria, tetanus and pertussis (DTP), measles, polio and bacille Calmette-Guérin (BCG) vaccines. As EPI-SA evolved, new vaccines were introduced, such as the hepatitis B vaccine in 1995 and Haemophilus influenzae type B vaccine in 1999. Since then, pneumococcal vaccine (PCV) and rotavirus vaccine were introduced in 2009, while human papillomavirus (HPV) vaccine was introduced in 2014. ${ }^{[1]}$

NAGI plays an instrumental role in the introduction of several new vaccines to the NIP but also to related matters, such as expanding immunisation services to all age groups and vaccination during the COVID-19 crisis. $^{[1,7]}$ Among other successes of EPI-SA are elimination of neonatal tetanus in 2002 and the country being declared polio free in 2006. ${ }^{[8]}$ The evolution of SA's immunisation landscape brings with it challenges that require use of locally available evidence to strengthen the NIP. A key area of SA's NIP that requires strengthening is improvement of vaccination coverage.

High-quality, relevant and timely national evidence is needed to inform immunisation practices and policies aimed at effective control of VPDs. The evidence needed is generated from diverse initiatives that include research. Research in vaccines and immunisation plays an important role in shaping immunisation policies at national level. It is therefore important to take stock of the research landscape involving vaccines and immunisation in SA.

\section{Objectives}

The objective of this study was to characterise human vaccine and immunisation research in SA by means of: (i) the most published journals; (ii) trends in the number of studies published annually; (iii) trends in the type of studies published; (iv) the most researched diseases; and $(v)$ the affiliation of the first authors of the relevant studies.

\section{Methods}

\section{Search strategy}

We conducted a bibliometric study. Seven electronic databases (PubMed; Scopus; Web of Science; Cochrane; CINAHL; Africa-Wide Information; and MEDLINE (via EBSCOhost)) were searched for eligible studies published in English between 1 January 2007 and 31 March 2020. We chose 2007 - 2020, as the period was characterised by rapid developments in vaccinology and the introduction of new vaccines into the NIP. A combination of medical subheadings $(\mathrm{MeSH})$ and free search terms for vaccines, vaccination, immunisation and SA were used. The full search strategy that was used in PubMed is shown in Table 1.

\section{Study selection}

Studies were included if they: (i) were conducted in humans; (ii) were conducted in SA; and (iii) focused on vaccines, immunisation programmes, policies or epidemiology of VPDs targeted by traditional or new vaccines. Studies reporting the epidemiology of VPDs without any reference to a specific vaccine were excluded, while only primary studies were included. All types of reviews were excluded.

Following the search, all retrieved articles were uploaded into EndNote V9 (Clarivate Analytics, USA), where duplicates were removed. The articles were then exported from EndNote to Rayyan (Qatar Computing Research Institute, Qatar), where the titles and abstracts were screened. ${ }^{[9]}$ The articles were screened by one reviewer (MIM) and verified by a second reviewer (ECH). Disparities that arose during the review process were resolved by discussion and consensus by inviting one of the co-authors to serve as a third reviewer.

\section{Data extraction and analyses}

Data from included articles were extracted by one reviewer (MIM) using a predesigned data extraction form, and verified by a second reviewer (ECH). Extracted data consisted of the study period, type of study (clinical or operational), class of study, targeted vaccine, disease and population, as well as the affiliation of the first author, i.e. listed as ' 1 '. Information regarding journal impact factor (JIF) was extracted. Extracted data were then imported into STATA version 14 (StataCorp., USA) for analysis. Results were presented as proportions.

The number of publications per journal was tabulated. Journals were ranked based on the number of published studies. In the event of a tie, journals were ranked in descending order using their latest JIF retrieved from Clarivate Analytics (Clarivate, USA). Included studies were stratified by affiliations (academic institutions) of the authors.

\section{Results}

Our search yielded 9212 articles: 1216 from PubMed; 416 from Cochrane; 4310 from EBSCOhost; 1825 from Scopus; and 1445 from Web of Science. Of these 9212 articles, 24 were identified through alerts from the relevant databases.

Using EndNote and Rayyan, 3631 duplicates were removed, leaving 5581 studies for screening. Based on titles and abstracts, 638 articles

\footnotetext{
Table 1. A summary of the search query

\#1 Vaccine (MeSH) OR Immuni ${ }^{\star}(\mathrm{MeSH})$ OR Vaccination (MeSH) OR 'Immuni Schedule' OR 'Immuni Program' OR 'Expanded Programme on Immuni^’ OR EPI OR Vaccinate OR 'Mass Vaccination’ OR 'Routine Vaccination' OR ‘School Based Vaccination' OR 'Outreach Vaccination' OR 'Supplementary Vaccination' OR 'Catch up Vaccination' OR 'Vaccine Trial' OR 'Vaccine Acceptance' OR 'Vaccine Hesitancy' OR 'Vaccine Induced Immunity' OR Immunogenicity OR 'Immune Response' OR 'Vaccination Coverage' OR 'Vaccine Knowledge'

\#2 'Republic of South Africa' OR 'South Africa'

\#3 \#1 AND \#2

Filters $\quad$ Publication year: 1 January 2007 - 31 December 2019; Language: English; Population: Human; Geographical focus: South Africa

Search date 21 June 2019 updated via alerts till 31 March 2020
} 
were retained for full-text assessment. Of these, 366 met the inclusion criteria. The literature screening and selection process are shown in Fig. 1, using the Preferred Reporting Items for Systematic Reviews and Meta-Analyses (PRISMA) statement. ${ }^{[10]}$

\section{Characteristics of included studies}

The 366 included studies were published in 101 peer-reviewed journals. The highest number of studies was published in Vaccine $(n=75 ; 20.5 \%)$, followed by SAMJ $(n=30$; $8.2 \%)$ and PLoS ONE ( $n=24 ; 6.6 \%)$. Of the 366 studies included, 54 (14.8\%) were published in 10 local (SA) journals and 312 $(85.2 \%)$ in international (non-SA) journals. Table 2 shows the top 10 journals where the included studies were published. The corresponding JIF is indicated.
Trends in the number of published studies, 2007 - 2019

In 2007, only 13 vaccine and immunisation research articles were published compared with 47 studies in 2015. A steady increase in the number of published studies was observed between 2009 and 2013. We unexpectedly observed a steady decline in the number of published studies from 2015 $(n=47)$ to $2017(n=27)$, followed by a sudden increase to 37 in 2019 (Fig. 2). In the first quarter of 2020, we identified 5 published studies (data not shown on the graph).

Trends in the types of human vaccine and immunisation studies in SA, 2007 - 2019

Trends in types of studies over time From 2007 to 2009, we observed several

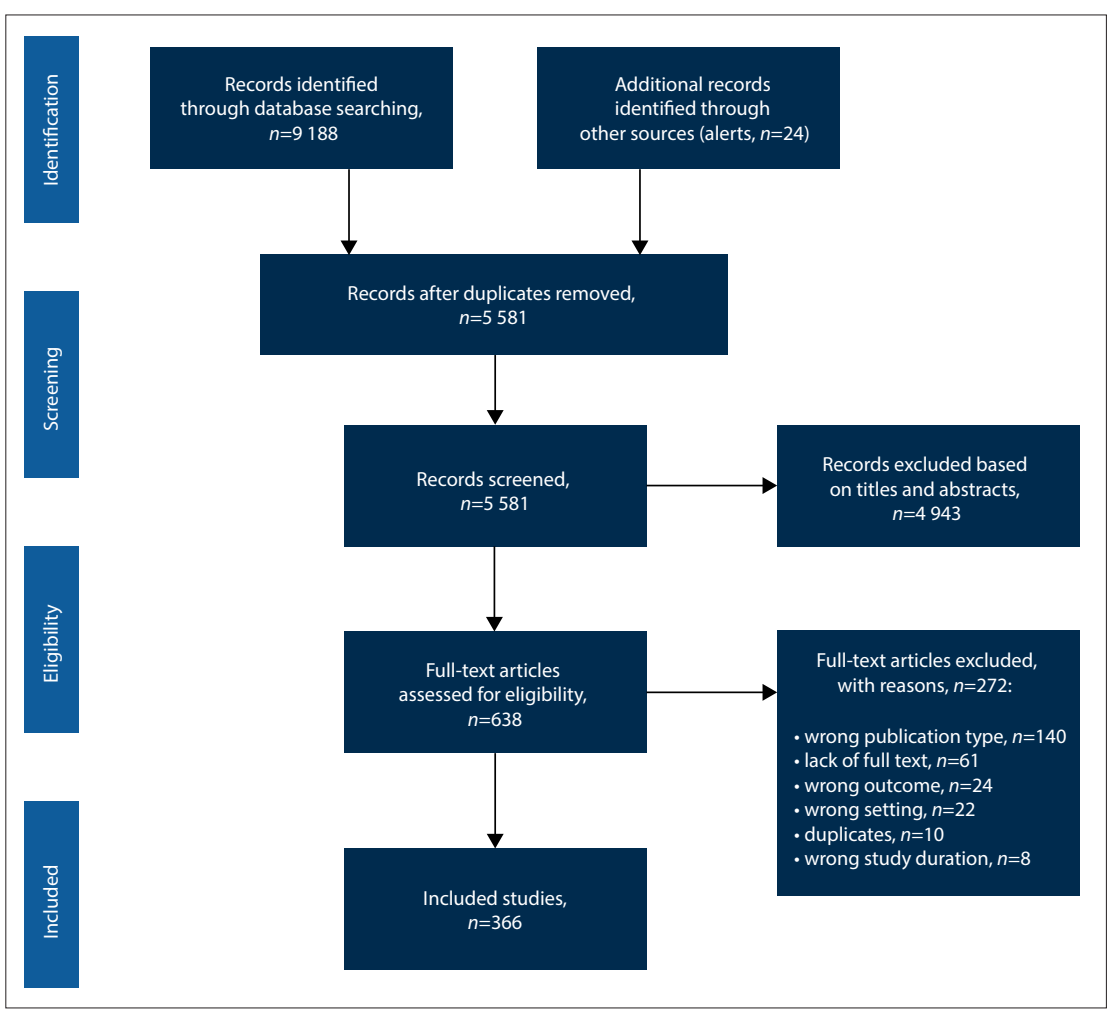

Fig. 1. Preferred Reporting Items for Systematic Reviews and Meta-Analyses (PRISMA) flow diagram. knowledge, attitude and practice (KAP) $(n=7 ; 17.1 \%)$, burden of disease (BOD) $(n=6$ $14.6 \%)$ and advocacy $(n=8 ; 19.5 \%)$ studies. In 2010, the largest number of KAP $(n=8$; $44.4 \%)$ and vaccine-coverage studies $(n=6$; $33.33 \%)$ were published in a single year. Additionally, we observed an increase in clinical trials $(n=6 ; 33.33 \%)$. The trend in clinical trials remained steady from 2010 to 2019 ( $n=6-8)$, except in 2014 ( $n=14 ; 41.1 \%)$ and 2017 ( $n=11 ; 40.7 \%)$, during which a large proportion of studies was published. Advocacy studies occurred most frequently during $2015(n=12 ; 25.5 \%)$ and $2018(n=8$; $25 \%)$. The number of immunology studies remained between 1 and 3 annually, except for $2013(n=4 ; 10.5 \%), 2015(n=5 ; 10.6 \%)$ and $2016(n=4 ; 12.1 \%)$ (Fig. 3$)$.

\section{Classification of included studies Type}

Operational studies ( $n=189 ; 51.7 \%)$ were published relatively more often than clinical studies ( $n=177 ; 48,3 \%$ ). Among the operational studies, most were KAP studies ( $n=57 ; 30.1 \%)$, followed by studies on advocacy $(n=52 ; 27.5 \%)$ and vaccine coverage $(n=23 ; 12.2 \%)$. For clinical studies, the largest outputs were clinical trials $(n=88 ; 49.7 \%)$ and BOD studies $(n=56 ; 31.6 \%)$ (Table 3).

\section{Disease}

A total of 318 (86.9\%) of the studies focused on a single disease. Studies focusing on HIV made the largest individual contribution to the relevant research outputs $(n=75 ; 23.6 \%)$, with HPV $(n=63 ; 19.8 \%)$ and tuberculosis (TB) $(n=61 ; 16.7 \%)$ comprising the second and third largest contributions, respectively. Forty-eight studies involved multiple VPDs covered in EPI-SA (Table 4).

\section{Study population}

One hundred and twenty-two (33.3\%) studies focused on childhood-only immunisation, followed by adult-only immunisation $(n=88$; $24 \%)$. Studies involving adolescent-only

Table 2. Top 10 journals where the included studies were published, $N=182$

\begin{tabular}{lll}
\hline Journal & Journal impact factor, 2018 & Studies, $\boldsymbol{n}$ (\%) \\
\hline Vaccine & 3.269 & $75(20.5)$ \\
South African Medical Journal (SAMJ) & 1.5 & $30(8.2)$ \\
PLoS ONE & $2.776(6.6)$ & $12(3.3)$ \\
Paediatric Infectious Diseases Journal & 2.456 & $8(2.2)$ \\
Journal of Infectious Diseases & 5.045 & $8(2.2)$ \\
Human Vaccines and Immunotherapeutics & 2.592 & $7(1.9)$ \\
Clinical Infectious Diseases & 9.055 & $6(1.6)$ \\
New England Journal of Medicine (NEJM) & 70.670 & $6(1.6)$ \\
Expert Review of Vaccines & 4.551 & $6(1.6)$ \\
International Journal of Infectious Diseases & 3.538 &
\end{tabular}




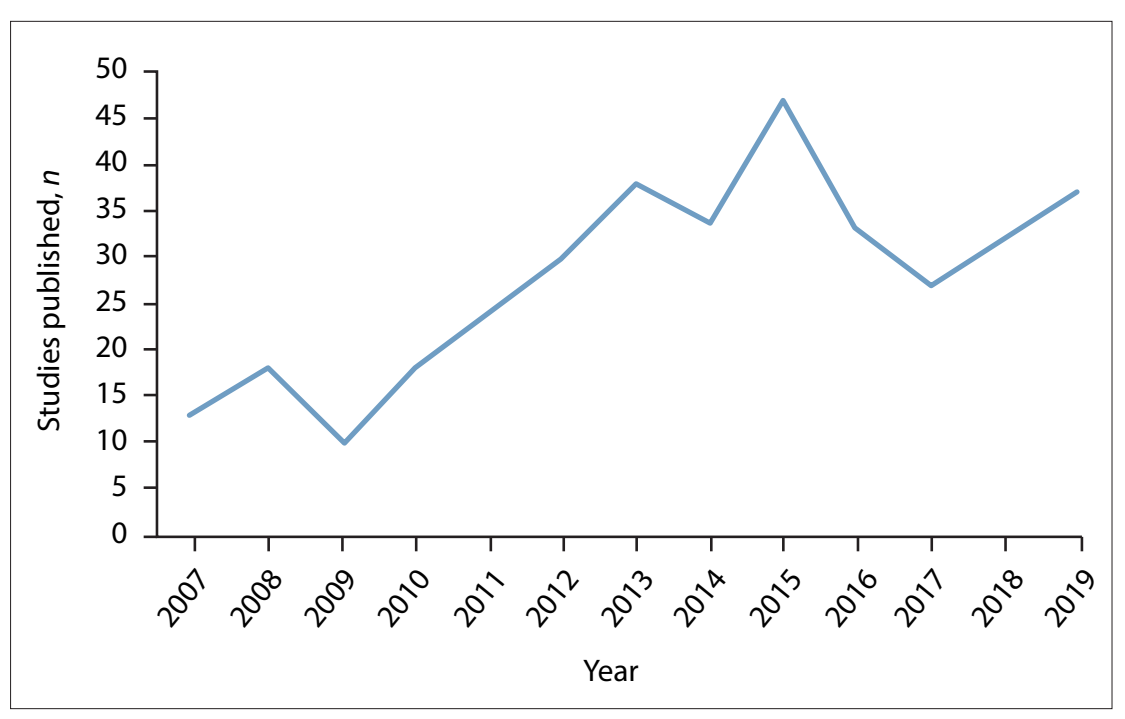

Fig. 2. Trend in the number of published vaccine and immunisation research studies in South Africa from 2007 to 2019.

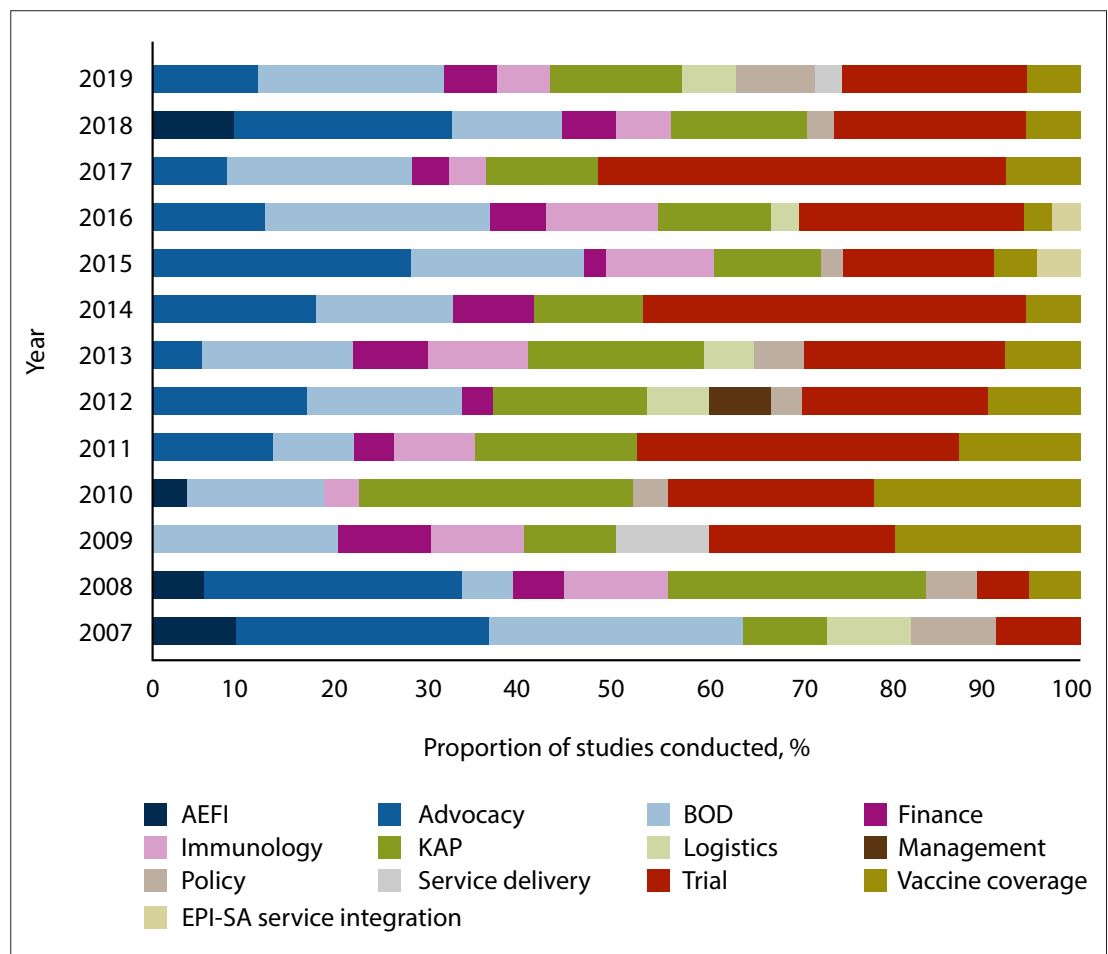

Fig 3. Trend in the types of human vaccine and immunisation studies in South Africa from 2007 to 2019. $(A E F I=$ adverse events following immunisation; $B O D=$ burden of disease; $K A P=$ knowledge, attitude and practice; EPI-SA = Expanded Programme on Immunisation in South Africa.)

immunisation accounted for just 26 (7.1\%) of all studies reviewed. The remaining 130 (35.5\%) studies involved multiple age groups (Fig. 4).

\section{Affiliation of first authors of vaccine and immunisation studies \\ Of the 366 studies, the first authors of 256 studies were affiliated with 28 SA institutions. Of the 256 SA-affiliated studies, 200 were affiliated with 10 uni-}

\section{Discussion}

We successfully identified and profiled research outputs on human vaccines and immunisation in SA between 2007 and 2019. Our key findings were as follows: $(i)$ research outputs on human vaccines and immunisation are published in both SA (local) journals and non-SA (international) journals; (ii) there was a steady increase in the number of publications on human vaccine and immunisation research outputs in SA over the period under review; (iii) clinical trials, BOD and KAP studies dominated the published research outputs during the study period; (iv) human vaccine and immunisation research outputs in SA are conducted across all age groups, with more focus on children; and $(v)$ the most represented authors of the identified research outputs were from the universities of Cape Town and the Witwatersrand, Johannesburg.

Journal altmetrics are routinely considered by researchers when choosing a journal in which to publish. ${ }^{[1]}$ Other reasons for choosing to publish in certain journals are the journal's rate of acceptance and interest in the study. This is important, because journal acceptance rates are widely recognised to be limited. Therefore, authors often require multiple submissions prior to being published. Given a choice, in addition to the scope, most researchers would opt to publish their research in a journal with a high JIF, such as NEJM. ${ }^{[12]}$ Of note is that only $54(14.8 \%)$ studies were published in SA journals and 310 (85.2\%) in international journals. However, of the top 10 journals where the research outputs of interest were published, SAMJ (local journal) had the lowest JIF (1.5) and yet, the journal ranked second. This is an encouraging finding, given that local journals are a useful source of local evidence.

Impressively, a steady increase in research outputs was observed between 2007 and 2015. This period coincided with the introduction of new vaccines by EPI-SA, such as those against rotavirus, pneumococcal disease, pertussis and HPV. ${ }^{[1]}$ When we assessed the research outputs by topics, HPV, pneumococcal disease and rotavirus were among the top 6 . This may suggest that the introduction of new vaccines could have enhanced research on topics related to these vaccines. There was an unexpected sharp decline in publication outputs in 2016 and 2017, despite an increase in gross expenditure on research and development in SA. ${ }^{[13]}$ Our speculation in this regard is that absence of new vaccine introduction during 2015 2019 may partly explain the decline. This is evident in the case of rotavirus and HPV, 


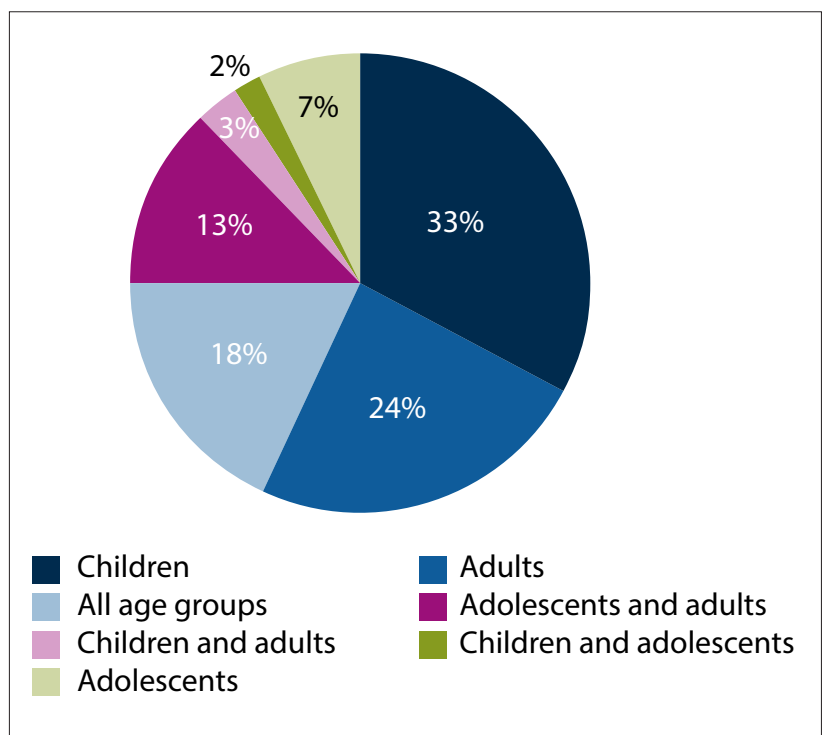

Fig. 4. Distribution of South African vaccine and immunisation research per targeted age group.

Table 3. Classification of the types of vaccine and immunisation studies, $N=366$

\begin{tabular}{ll}
\hline Type of study & Studies, $\boldsymbol{n}(\%)$ \\
\hline Clinical & $177(48.3)$ \\
Clinical trials (phase $1-4)$ & $88(49.7)$ \\
Burden of disease & $58(32.7)$ \\
Immunology & $25(14.1)$ \\
AEFI & $6(3.4)$ \\
Operational & $189(51.7)$ \\
Knowledge attitude and practice & $57(30,1)$ \\
Advocacy & $56(29.6)$ \\
Vaccine coverage & $23(12.2)$ \\
Finance & $19(10.1)$ \\
Logistics & $15(7.9)$ \\
Policy & $11(5.8)$ \\
EPI-SA service integration & $4(2.1)$ \\
Service delivery & $2(1.05)$ \\
Management & $2(1.05)$ \\
AEFI = adverse events following immunisation; EPI-SA = Expanded Programme on \\
Immunisation in South Africa.
\end{tabular}

which were introduced into EPI-SA in 2014. During this period, we observed the following trends: HPV studies were mostly published between 2013 and 2015 ( $n=27$ ), with a decrease to 3 studies published between 2016 and 2017, and an increase to 17 studies published between 2018 and 2019. The trend in rotavirus-related publications follows a similar pattern, with 11 studies published between 2012 and 2015 and 4 studies published between 2016 and 2019.

Another factor worth noting is the high number of advocacy studies published in 2015 ( $n=11)$, with a subsequent decrease in the following years, ranging from a low of 2 in 2017 to a high of 7 in 2018 . We posit that this was potentially instrumental towards the increase in gross expenditure on research. Interestingly, we observed a sharp increase in publication outputs between 2018 and 2019. We attribute the increase to the need for improving awareness and knowledge on immunisation as a result of recurrent outbreaks of VPDs, such as measles. ${ }^{[14]}$ A large proportion of research outputs published in 2018
Table 4. Published studies reported by type of the disease,

\begin{tabular}{ll}
$\boldsymbol{N}=\mathbf{3 6 6}$ & Studies, $\boldsymbol{n}$ (\%) \\
\hline Disease & $75(23.6)$ \\
HIV & $63(19.8)$ \\
Human papillomavirus & $61(16.7)$ \\
Tuberculosis & $48(13.1)$ \\
Multiple VPDs with EPI-SA & $30(8.2)$ \\
Influenza-related illness & $30(8.2)$ \\
Pneumococcal disease & $17(4.6)$ \\
Rotavirus & $14(3.8)$ \\
Hepatitis B virus & $7(1.9)$ \\
Measles & $6(1.6)$ \\
Streptococcal disease & $4(1.1)$ \\
Polio & $3(0.8)$ \\
Neisserria meningitidis & $2(0.6)$ \\
Haemophillus influenza type B & $2(0.6)$ \\
Rubella & $2(0.6)$ \\
Hepatitis A virus & $1(0.3)$ \\
Malaria & $1(0.3)$ \\
Pertussis & \\
vPDs = vaccine preventable diseases; EPI-SA = Expanded Programme on Immunisation \\
in South Africa.
\end{tabular}

and 2019 was focused on KAP and advocacy concerning vaccines. For example, 19 of the 32 studies published in 2018 were operational studies, of which 12 focused on KAP and vaccine advocacy in SA. Published studies on BOD and KAP featured prominently, which is a positive finding, given that both types of studies are critical in guiding immunisation practices and policies in SA. KAP studies were mainly related to HIV vaccine trials and HPV vaccination programmes. Studies included also focused on catch-up vaccinations related to HPV to prevent cervical cancer in adult women, and on measles to monitor the effect of mass immunisation activities following the measles outbreak of 2009 - 2011. Admirably, the types of studies most published appeared to align with the DoH's strategic plan for 2015 2020, which emphasises the need to promote health and prevent and reduce the disproportionate burden of disease, especially the HIV and TB epidemic. ${ }^{[15]}$

Regarding the types of research that were most published, clinical trials topped the list. SA is endemic for TB and HIV, the duo of epidemics that puts considerable pressure on the country's health system. ${ }^{[16]}$ Given that new vaccines against TB and HIV epidemics are a public health priority, the country is at the forefront of guiding the development and testing of candidate vaccines against these diseases. ${ }^{[17]}$ Also worth noting is that the identified research outputs were conducted in all population groups, i.e. children, adolescents and adults. This is an encouraging finding as life-course vaccination (vaccinating all age groups) has been identified as a key strategy to optimise the benefits of vaccines. ${ }^{[18]}$

Affiliations of authors give an indication of institutions at the forefront of vaccine and immunisation research in SA. First authors of most of the identified research outputs were affiliated with the universities of Cape Town and the Witwatersrand. This is not a surprising observation, as these are the top two ranked institutions in the country by the Times Higher Education world university rankings. ${ }^{[19]}$

Furthermore, the presence of established vaccine research units in specific organisations or universities contributes towards research outputs. It is therefore no surprise that institutions such as the 
universities of Cape Town and the Witwatersrand were observed to be at the forefront of vaccine-related research.

The affiliations of co-authors could also suggest the scope of research collaborations in the field of vaccine and immunisation research, although this was not explored as part of the current study.

\section{Study limitations}

This bibliometric study has some limitations that are worth noting in the context of our findings. Systematic reviews, meta-analyses and guidelines were not considered for inclusion, as we focused on primary studies. It is generally recognised that evidence syntheses and guidelines are essential in shaping immunisation practices and policies. Grey literature and non-English articles were also not included, which may have resulted in the exclusion of potentially relevant data.

To further improve the publication of research in local journals, we propose that researchers should be incentivised and encouraged. For example, incentivisation could be done through weighting of researchers' contributions to the local research publication when applying for local funding, as well as academic promotions.

\section{Conclusions}

Our findings summarise the types and trends of human vaccine and immunisation research in SA. To our knowledge, this summary has been lacking, except for a literature review conducted as part of a larger study. ${ }^{[2]}$ Our study improved on this by restricting included studies to human vaccine-related work and using a systematic review methodology, thus producing evidence that more accurately reflects the trends and types of human vaccine and immunisation research in SA. Our findings show a similar profile of vaccine and immunisation research reported previously at a regional level..$^{[5,6]}$ These findings should be used to inform and support immunisation policies and practices locally by highlighting gaps in key areas of immunisation research that need to be better addressed to ensure that immunisation policies and practices are guided by locally relevant and contextspecific evidence.

Declaration. None.

Acknowledgements. None.

Author contributions. This study was conceived by BMK, GDH and RM.
MIM and ECH conducted the literature search and screening. Relevant data were extracted by MIM, with oversight from ECH, EAD and BMK. MIM developed the first draft of the manuscript, which was finalised with input from all co-authors.

Funding. None.

Conflicts of interest. None.

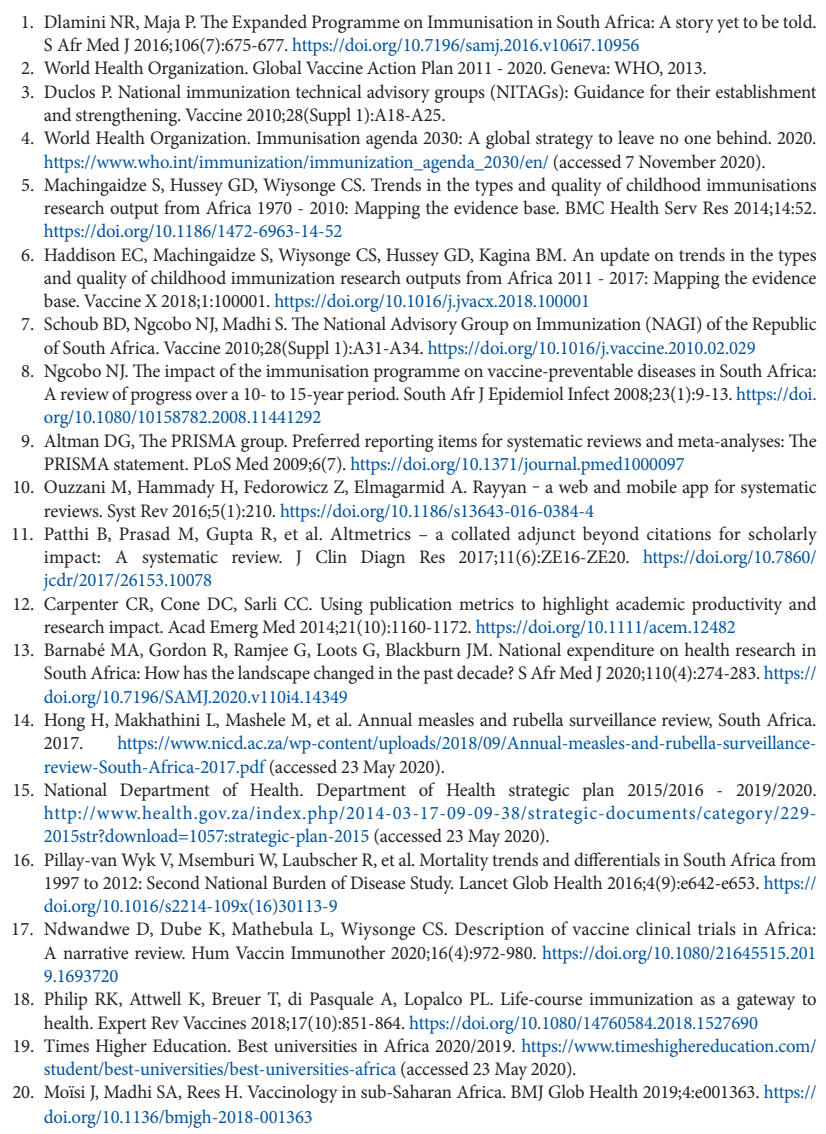
and quality of childhood immunization research outputs from Africa 2011 - 2017: Mapping the evidence base. Vaccine X 2018;1:100001. https://doi.org/10.1016/j.jvacx.2018.100001

7. Schoub BD, Ngcobo NJ, Madhi S. The National Advisory Group on Immunization (NAGI) of the Republic of South Africa. Vaccine 2010;28(Suppl 1):A31-A34. https://doi.org/10.1016/j.vaccine.2010.02.029

8. Ngcobo NJ. The impact of the immunisation programme on vaccine-preventable diseases in South Africa: A review of progress over a 10- to 15-year period. South Afr J Epidemiol Infect 2008;23(1):9-13. https://doi. org/10.1080/10158782.2008.11441292

9. Altman DG, The PRISMA group. Preferred reporting items for systematic reviews and meta-analyses: The PRISMA statement. PLoS Med 2009;6(7). https://doi.org/10.1371/journal.pmed1000097

10. Ouzzani M, Hammady H, Fedorowicz Z, Elmagarmid A. Rayyan - a web and mobile app for systematic reviews. Syst Rev 2016;5(1):210. https://doi.org/10.1186/s13643-016-0384-4

11. Patthi B, Prasad M, Gupta R, et al. Altmetrics - a collated adjunct beyond citations for scholarly impact: A systematic review. J Clin Diagn Res 2017;11(6):ZE16-ZE20. https://doi.org/10.7860/ jcdr/2017/26153.10078

12. Carpenter CR, Cone DC, Sarli CC. Using publication metrics to highlight academic productivity and research impact. Acad Emerg Med 2014;21(10):1160-1172. https://doi.org/10.1111/acem.12482

13. Barnabé MA, Gordon R, Ramjee G, Loots G, Blackburn JM. National expenditure on health research in South Africa: How has the landscape changed in the past decade? S Afr Med J 2020;110(4):274-283. https:// doi.org/10.7196/SAMJ.2020.v110i4.14349

14. Hong H, Makhathini L, Mashele M, et al. Annual measles and rubella surveillance review, South Africa. 2017. https://www.nicd.ac.za/wp-content/uploads/2018/09/Annual-measles-and-rubella-surveillancereview-South-Africa-2017.pdf (accessed 23 May 2020).

15. National Department of Health. Department of Health strategic plan 2015/2016 - 2019/2020. http://www.health.gov.za/index.php/2014-03-17-09-09-38/strategic-documents/category/2292015str?download=1057:strategic-plan-2015 (accessed 23 May 2020).

16. Pillay-van Wyk V, Msemburi W, Laubscher R, et al. Mortality trends and differentials in South Africa from 1997 to 2012: Second National Burden of Disease Study. Lancet Glob Health 2016;4(9):e642-e653. https:// doi.org/10.1016/s2214-109x(16)30113-9

17. Ndwandwe D, Dube K, Mathebula L, Wiysonge CS. Description of vaccine clinical trials in Africa: A narrative review. Hum Vaccin Immunother 2020;16(4):972-980. https://doi.org/10.1080/21645515.201 A narrative

18. Philip RK, Attwell K, Breuer T, di Pasquale A, Lopalco PL. Life-course immunization as a gateway to health. Expert Rev Vaccines 2018;17(10):851-864. https://doi.org/10.1080/14760584.2018.1527690

19. Times Higher Education. Best universities in Africa 2020/2019. https://www.timeshighereducation.com/ student/best-universities/best-universities-africa (accessed 23 May 2020).

20. Moisi J, Madhi SA, Rees H. Vaccinology in sub-Saharan Africa. BMJ Glob Health 2019;4:e001363. https:// doi.org/10.1136/bmigh-2018-001363 